\title{
GRAPHS OF MEASURABLE FUNCTIONS
}

\author{
J. J. BUCKLEY
}

Abstract. Necessary and sufficient conditions are given for the measurability of a function in terms of its graph.

1. Introduction. Let $X_{1}$ be an arbitrary nonempty set and let $\mathscr{A}$ be a $\sigma$-algebra of subsets of $X_{1} . X_{2}$ is a complete separable metric space and $\mathscr{B}_{2}$ is the Borel field in $X_{2}$. The Borel field is the minimal $\sigma$-algebra over the open sets. It $X_{1}$ is a topological space, then $\mathscr{B}_{1}$ is the Borel field in $X_{1}$. If $f: X_{1} \rightarrow X_{2}$, then $G(f)=\left\{(x, y) \mid x \in X_{1}, y=f(x)\right\}$ and $f$ is $\mathscr{B}_{1}(\mathscr{A})$-measurable if and only if $f^{-1}(O) \in \mathscr{B}_{1}(\mathscr{A})$ for every $O$ open in $X_{2}$. It is known that: (1) if $X_{2}=R$ and if $f$ is $\mathscr{A}$-measurable, then $G(f) \in \mathscr{A} \times \mathscr{B}_{2}$, the product $\sigma$-algebra [2, p. 143]; and (2) if $X_{1}$ is a complete separable metric space, then $f$ is $\mathscr{B}_{1}$-measurable if and only if $G(f)$ is a Borel subset of $X_{1} \times X_{2}$ (Propositions 1 and 6 in §3). This characterization of Borel measurability has been used to define measurability of set-valued maps [1]. The following theorem gives the corresponding result to (2) for Lebesgue measurable functions. For completeness, the propositions on Borel and analytic sets needed to prove the Theorem and to establish (2) above are included in $\S 3$ on analytic sets which follows the proof of the Theorem.

2. Main result. Let $\mu$ be a measure on $\mathscr{B}_{1}$ and let $\bar{\mu}$ be the completion of $\mu$ and $\mathscr{A}$ the completion of $\mathscr{B}_{1}$. Let $\mathscr{N}$ be all the sets of $\bar{\mu}$ measure zero.

TheOREM. Let $X_{1}$ be a complete separable metric space. Then $f$ is $\mathscr{A}$ measurable if and only if $G(f) \in \mathscr{A} \times \mathscr{B}_{2}$.

Proof. a. Let $G(f) \in \mathscr{A} \times \mathscr{B}_{2}$.

1. If $E \in \mathscr{A} \times \mathscr{B}_{2}$, then there are sets $E_{1} \in \mathscr{B}_{1} \times \mathscr{B}_{2}, E_{2} \subset X_{1} \times X_{2}$, and $E_{3} \in \mathscr{N} \times \mathscr{B}_{2}$ (product $\sigma$-ring) such that $E=E_{1} \cup E_{2}$ and $E_{2} \subset E_{3}$, because if $\mathscr{S}$ is all such unions, then $\mathscr{S}$ contains all the measurable rectangles in $\mathscr{A} \times \mathscr{B}_{2}$ and $\mathscr{S}$ is a monotone class.

2. Each element in $\mathscr{B}_{1} \times \mathscr{B}_{2}$ is a Borel subset of $X_{1} \times X_{2}$.

3. Let $O$ be open in $X_{2}$ and let $G(f)=E_{1} \cup E_{2}, E_{2} \subset E_{3}$. Now $E_{3} \subset N \times B$, for some $N \in \mathscr{N}$ and some $B \in \mathscr{B}_{2}$, because if $\mathscr{T}$ is all subsets of $X_{1} \times X_{2}$

Received by the editors May 14, 1973.

AMS (MOS) subject classifications (1970). Primary 28A20.

Key words and phrases. Measurable sets, measurable functions, Borel measurable, Lebesgue measurable, analytic sets.

(c) American Mathematical Society 1974 
which are contained in a measurable rectangle in $\mathscr{N} \times \mathscr{B}_{2}$, then $\mathscr{T}$ is a $\sigma$-ring containing all the measurable rectangles in $\mathscr{N} \times \mathscr{B}_{2}$. Let $A_{i}=$ $\left(X_{1} \times O\right) \cap E_{i}, i=1,2,3$. If $P$ is the projection of $X_{1} \times X_{2}$ into $X_{1}$, then $f^{-1}(O)=P\left(A_{1}\right) \cup P\left(A_{2}\right)$. Now $P\left(A_{2}\right) \subset P\left(A_{3}\right) \subset N$, so $P\left(A_{2}\right) \in \mathscr{A}$.

4. We show that $P\left(A_{1}\right) \in \mathscr{A}$, and it follows that $f$ is $\mathscr{A}$-measurable. Now $P\left(A_{2}\right)$ is contained in some $C \in \mathscr{B}_{1}$ of $\bar{\mu}$ measure zero. Let $D=$ $X_{1}-C$ and let $\bar{D}$ be the closure of $D$. Define $g: X_{1} \rightarrow X_{2}$ so that its graph is $\left(E_{1} \cap\left(D \times X_{2}\right)\right) \cup\left(C \times\left\{y_{0}\right\}\right)$, some fixed $y_{0} \in X_{2}$. Let $g^{1}$ be $g$ restricted to $\bar{D}$. Let $A_{4}=(D \times O) \cap E_{1}$ and let $A_{5}=(\bar{D} \times O) \cap G\left(g^{1}\right)$. Since $\bar{D}$ is a complete separable metric space we have that $P\left(A_{5}\right)$ and $\bar{D}-P\left(A_{5}\right)=$ $P\left(\left(\bar{D} \times\left(X_{2}-O\right)\right) \cap G\left(g^{1}\right)\right)$ are analytic subsets of $\bar{D}$ (Proposition 3$)$. Therefore $P\left(A_{5}\right)$ is a Borel subset of $\bar{D}$ (Proposition 5). So there is an $F \in \mathscr{B}_{1}$ so that $P\left(A_{5}\right)=F \cap \bar{D}$. Then $P\left(A_{4}\right)=F \cap D$ and $P\left(A_{4}\right)$ is a Borel subset of $X_{1}$. But $P\left(A_{1}\right)=P\left(A_{4}\right) \cup\left(P\left(A_{1}\right) \cap C\right)$ and $P\left(A_{1}\right) \cap C \in \mathscr{N}$, so that $P\left(A_{1}\right) \in \mathscr{A}$.

b. Let $f$ be $\mathscr{A}$-measurable. For each $n$ choose a disjoint sequence $\left\{A_{i, n}\right\}_{i=1}^{\infty}$ of elements in $\mathscr{B}_{2}$ whose union is $X_{2}$ and whose diameter is less than $1 / n$. Then

$$
G(f)=\bigcap_{n=1}^{\infty} \bigcup_{i=1}^{\infty}\left(f^{-1}\left(A_{i, n}\right) \times A_{i, n}\right) \in \mathscr{A} \times \mathscr{B}_{2},
$$

because $f^{-1}\left(\mathscr{B}_{2}\right) \subset \mathscr{A}$.

Corollary. Let $f: R^{n} \rightarrow R$, let $\mathscr{A}$ be the Lebesgue subsets of $R^{n}$, and let $\mathscr{B}$ be the Borel subsets of $R$. Then $f$ is Legesgue measurable if and only if its graph is measurable with respect to the product $\sigma$-algebra $\mathscr{A} \times \mathscr{B}$.

3. Analytic sets. If $T$ is a topological space, then the analytic subsets of $T$ are the continuous images of the Borel subsets of $T . X_{1}$ and $X_{2}$ are complete separable metric spaces, $I$ is the irrational numbers in $(0,1)$ with the usual topology, $N$ is the positive integers (discrete topology), and $d_{2}$ is the metric on $X_{2}$.

Proposition 1. If $f: X_{1} \rightarrow X_{2}$ is $\mathscr{B}_{1}$-measurable, then $G(f)$ is a Borel subset of $X_{1} \times X_{2}[3, p p .384,457]$.

Proof. The function $h\left(x_{1}, x_{2}\right)=d_{2}\left(f\left(x_{1}\right), x_{2}\right)$ is a Borel measurable mapping of $X_{1} \times X_{2}$ into the reals. Hence $G(f)=h^{-1}(0)$ is Borel.

Proposition 2. Every Borel subset of $X_{1}$ is the continuous image of $I$ $[3, p .446]$.

Proof. Let $\mathscr{S}$ be all subsets of $X_{1}$ which are continuous images of $I$. Since every complete separable metric space is the continuous image of $I$ [3, p. 440], all the closed subsets of $X_{1}$ are in $\mathscr{S}$. Now $\mathscr{B}_{1}$ is the smallest class of subsets of $X_{1}$ which contains the closed sets and is closed under countable unions and intersections [3, p. 344]. It remains to show $\mathscr{S}$ is 
closed under countable unions and intersections. Let $A_{i}=f_{i}(I) \in \mathscr{S}$, $i=1,2, \cdots$, and let $A=\bigcup_{i=1}^{\infty} A_{i}, B=\bigcap_{i=1}^{\infty} A_{i}$. If $I_{n}$ is the irrationals in $(n-1, n), n \geqq 1$, define $f$ on $I^{*}=\bigcup_{n=1}^{\infty} I_{n}$ as follows: $f(x)=f_{n}(x-n+1)$ if $x \in I_{n}$. Then $f$ is continuous and $f\left(I^{*}\right)=A$. So, $A \in \mathscr{S}$ since $I^{*}$ is homeomorphic to $I$.

Define $E=\left\{x=\left(x_{1}, x_{2}, \cdots\right) \in I^{N} \mid f_{1}\left(x_{1}\right)=f_{2}\left(x_{2}\right)=\cdots\right\} . E$ is empty if and only if $B$ is empty. Define $f^{*}$ on $E$ as follows: $f^{*}(x)=f_{1}\left(x_{1}\right)$. Then $f^{*}$ is continuous and $f^{*}(E)=B$. Let $\bar{N}=N^{N}$. $\bar{N}$, with the usual metric, is a complete separable metric space. Since $I$ and $\bar{N}$ are homeomorphic [3, p. 407], there is a homeomorphism $\lambda: \bar{N}^{N} \rightarrow I^{N}$. Also, $\bar{N}^{N}$ is a complete separable metric space. Now $\lambda^{-1}(E)$ is closed in $\bar{N}^{N}$ because $E$ is closed in $I^{N}$ and so $\lambda^{-1}(E)=f(I), f$ continuous. Hence $B=f * \lambda f(I)$ and $B \in \mathscr{S}$.

Proposition 3. If $f: X_{1} \rightarrow X_{2}$ is continuous and $B \in \mathscr{B}_{1}$, then $f(B)$ is analytic [3, $p .454]$.

Proof. If $X_{2}$ is countable, then $f(B)$ is countable and in $\mathscr{B}_{2}$. Assume that $X_{2}$ is uncountable. Then there is a $G_{\delta}$-set $A$ in $X_{2}$ and a homeomorphism $g$ between $I$ and $A$ [3, p. 445]. By Proposition 2, $B=h(I), h$ continuous. So $f(B)=f h g(A)$ is analytic.

Proposition 4. If $A$ and $B$ are two disjoint analytic subsets of $X_{1}$, then there is a Borel set $E \subset X_{1}$ so that $A \subset E$ and $E \cap B$ is empty [3,p. 485].

Proposition 5. If $A$ and $A^{c}$ are analytic subsets of $X_{1}$, then $A$ is a Borel set $[3, p .486]$.

Proof. Let $B=A^{c}$ in Proposition 4.

Proposition 6. If $f: X_{1} \rightarrow X_{2}$ such that $G(f)$ is a Borel subset of $X_{1} \times X_{2}$, then $f$ is $\mathscr{B}_{1}$-measurable [3, p. 489].

Proof. Let $O$ be open in $X_{2}$. By Proposition $3, f^{-1}(O)$ is analytic in $X_{1}$ because it is the projection of Borel $G(f) \cap\left(X_{1} \times O\right)$ into $X_{1}$. Similarly, $\left(f^{-1}(O)\right)^{c}=f^{-1}\left(O^{c}\right)$ is analytic in $X_{1}$. Therefore $f^{-1}(O)$ is Borel by Proposition 5 .

\section{REFERENCES}

1. R. J. Aumann, Integrals of set-valued functions, J. Math. Anal. Appl. 12 (1965), 1-12. MR 32 \#2543.

2. P. R. Halmos, Measure theory, Van Nostrand, Princeton, N.J., 1950. MR 11, 504.

3. K. Kuratowski, Topologie. Vol. I, 2nd ed., Monografie Mat., Tom 20, WarszawaWroclaw, 1948; English transl., new ed., rev. and aug., Academic Press, New York; PWN, Warsaw, 1966. MR 10, 389; 36 \#840.

Department of Mathematics, University of South Carolina, Columbia, South CAROLINA 29208 